\title{
LA PROFESIÓN DOCENTE Y CALIDAD DE LA EDUCACIÓN: DESAFÍOS PARA LA FORMACIÓN DEL DOCENTE ECUATORIANO
}

\author{
The Educational Profession and Quality of the Education: Challenges for the \\ Ecuadorian Formation of the Educational
}

\author{
Blanca Cortón Romero, Dra. C. \\ Universidad de Oriente, Cuba \\ https://ORCID.org/0000-0002-0144-2228 \\ blancacr@uo.edu.cu
}

\author{
Julia Esther Céspedes Acuña, Dra. C. \\ Universidad de Oriente, Cuba \\ https://ORCID.org/0000-0003-1467-1393 \\ estherca@uo.edu.cu
}

\author{
Rosangela Caicedo Quiroz, Dra. C. \\ Instituto Superior Tecnológico \\ Bolivariano de Tecnología, Ecuador \\ https://orcid.org/0000-0003-0737-9132 \\ rcaicedo@bolivariano.edu.ec
}

Palabras claves: Calidad de la Educación, Formación Docente, Rol Profesional del Docente, Educación Ecuatoriana.

Keywords: Quality of the Education, Educational Formation, Professional List of the Educational One, Ecuadorian Education.
Recibido: 03 de marzo de 2021

Aceptado: 28 de abril de 2021

\section{RESUMEN}

La calidad de la educación constituye una preocupación de la comunidad internacional y gobiernos de todos los países. En Ecuador, la Ley Orgánica de Educación Intercultural de la República la que reconoce los principios generales de la actividad educativa y enfatiza en garantizar el derecho de las personas a una educación pertinente, adecuada, contextualizada, actualizada y articulada en todo el proceso educativo. Existen diversidad de enfoques y criterios sobre calidad de la educación, no obstante, se reconoce el papel y la importancia del maestro para el logro de la misma. Las autoras reflexionan sobre las transformaciones en la profesión docente y los desafíos para las instituciones formadoras respecto al mejoramiento continuo de la calidad de la educación ecuatoriana.

\section{ABSTRACT}

The quality of the education constitutes a concern of the international community and governments of all the countries. In Ecuador, the Organic Law of Education Intercultural of the Republic the one that recognizes the general principles of the educational activity and it emphasizes in guaranteeing the right from people to a pertinent, appropriate education, contextualizada, modernized and articulated in the whole educational process. Diversity of focuses and approaches exist it has more than enough quality of the education, nevertheless, it is recognized the paper and the teacher's importance for the achievement of the same one. The authors meditate on the transformations in the educational profession and the challenges for the institutions formadoras regarding the continuous improvement of the quality of the Ecuadorian education. 


\section{INTRODUCCIÓN}

El mejoramiento de la calidad de la formación de los profesionales de la educación constituye una exigencia de la sociedad contemporánea, un reto para las instituciones formadoras y una premisa para el perfeccionamiento de los sistemas educacionales y el éxito de las estrategias de desarrollo de los diferentes países. La significación de este aspecto se acrecienta en el caso de los países en desarrollo en los que la formación de los recursos humanos-proceso en el que los docentes desempeñan un papel fundamental- deviene un factor decisivo para el logro de sus objetivos estratégicos en pos del desarrollo.

Aunque existen diversidad de enfoques y criterios sobre calidad de la educación en general, y de la educación superior en particular, hay consenso con respecto al papel y la importancia del docente para el logro de la misma. Su decisivo papel, y los profundos cambios de la sociedad contemporánea que impactan en su rol profesional, le imponen importantes retos al proceso de formación de este profesional.

La importancia de la formación de docentes con un alto nivel de calificación ha sido reconocida por diversos organismos internacionales, entre ellos la Organización de Naciones Unidas (ONU). En el documento Transformar nuestro mundo: la Agenda 2030 para el Desarrollo Sostenible, aprobado por el organismo internacional en 2015, se incluyen entre los Objetivos de Desarrollo Sostenible (ODS) "Garantizar una educación inclusiva y equitativa de calidad y promover oportunidades de aprendizaje permanente para todos"; y se significa entre las metas cuyo logro debe contribuir al cumplimiento de dicho objetivo "... aumentar considerablemente la oferta de docentes calificados, incluso mediante la cooperación internacional para la formación de docentes en los países en desarrollo, especialmente los países menos adelantados y los pequeños Estados insulares en desarrollo"(ONU, 2015).

Independientemente de factores como la infraestructura, las reglamentaciones legales, los planes y programas de estudio y las demandas sociales, son los hombres y mujeres que diariamente se enfrentan a las aulas quienes pueden materializar los objetivos que garantizan la calidad de la educación; de ahí que, la formación de docentes sea considerada el elemento esencial en el mejoramiento continuo de los estándares de calidad de la educación por diversos estudiosos del tema, políticos y organizaciones nacionales, regionales e internacionales.

En el Informe del Grupo del Banco Mundial. Profesores excelentes: Cómo mejorar el aprendizaje en América Latina y el Caribe se señala "... la calidad de la educación está condicionada por la calidad de nuestros profesores..." y se define a estos como "....actores clave en los esfuerzos de la región por mejorar la calidad y los resultados educativos..." (Banco Mundial ,2014)

Entre los argumentos a partir de los cuales se sostiene la afirmación anterior se señala que "Los profesores no solo son actores clave de la producción de resultados de educación, sino también la parte interesada con más influencia en el proceso de reforma educativa... Debido a que gozan de una autonomía única cuando se cierran las puertas del aula, los profesores tienen un profundo poder de decisión en lo que respecta a qué políticas nuevas se pueden implementar con éxito..." (Banco Mundial ,2014)

Junto al reconocimiento del importante papel del docente en el logro de la calidad de la educación, hay también consenso en el reconocimiento a las dificultades y carencias que enfrenta el proceso de formación y el ejercicio de la profesión docente en América Latina y el Caribe, en este sentido el informe del Banco Mundial referido anteriormente plantea que "...Toda la evidencia disponible indica que la calidad de los profesores de América Latina y el Caribe es la limitación más importante al avance de la región hacia sistemas educativos de calidad internacional..." (Banco Mundial,2014). Se concuerda con este punto de vista, aunque se reconocen otros factores relacionados con las políticas públicas en general y educacionales en particular que inciden decisivamente en la calidad de la educación latinoamericana y caribeña.

Ecuador, enfrenta en la formación de docentes los retos comunes a todos los países de Latinoamérica y el mundo, consecuencia de las transformaciones en la sociedad contemporánea y su incidencia en las exigencias al rol profesional del docente, pero estos adquieren características peculiaridades que devienen de su situación económica, política y social particular; y en especial del estado real del proceso de formación docente.

El artículo que se presenta se propone reflexionar sobre las transformaciones en la profesión docente y los desafíos que se imponen a las instituciones formadoras en su relación con el mejoramiento continuo de la calidad de la educación ecuatoriana partiendo del análisis de documentos normativos de la sociedad y la educación ecuatoriana y de la experiencia pedagógica de los autores. 


\section{DESARROLLO}

\section{Formación de docentes y calidad de la educación superior}

En el análisis de los retos a la formación de docentes, en su vínculo con el mejoramiento continuo de la calidad de la educación en general y de la educación superior en particular resulta necesario partir de un posicionamiento teórico con respecto al contenido de los conceptos calidad de la educación y calidad de la educación superior, especificando en el caso de la sociedad ecuatoriana.

La definición de la calidad de la educación constituye un tema actual y polémico sobre el que existen posiciones y enfoques diversos en el contexto de América Latina y el Caribe entre los que se encuentran:

- Implica un juicio de valor respecto del tipo de educación que se quiere para formar un ideal de hombre y de sociedad a tono con las necesidades exigencias de hoy a nivel global y en cada país.

- Se identifica con eficiencia y eficacia a partir de considerar a la educación como un servicio existente en el mercado que tiene que satisfacer a sus usuarios.

- Se identifica solamente con los resultados de aprendizaje, e incluso de algunas materias como Matemáticas, Lenguas y Ciencias.

- Se relaciona con la capacidad para el desarrollo de los educandos a partir de que den sentido a lo que aprenden sobre la base de su utilidad para la vida.

- Enfoques críticos -en lo que se encuentran posiciones diversas- que centran la atención en el papel de la educación como preparación para el análisis crítico de la realidad como vía para contribuir al cambio social.

- Enfoque de derechos en educación, basado en los principios de gratuidad y obligatoriedad, y en los derechos a la no discriminación, la accesibilidad y la plena participación.

A juicio de los autores de este artículo el análisis de la calidad de la educación requiere de un enfoque integrado e integrador en el que se tengan en cuenta las diversas posiciones enunciadas y su contextualización a las peculiaridades específicas de cada sistema de educación.

Con respecto a la calidad de la educación superior durante un largo período de tiempo y hasta aproximadamente la década del 60 del pasado siglo XX, predominó una visión tradicional que privilegiaba aspectos como la tradición de la institución, la exclusividad de profesores y alumnos, y la disponibilidad de recursos materiales para el desarrollo del proceso formativo, al referirse a los principales indicadores de calidad, esta visión se correspondía con el criterio dominante sobre la relación universidad-sociedad en el que la primera era considerada como poseedora, y trasmisora absoluta de los conocimientos y la segunda como receptora pasiva y consumidora de dichos conocimientos.

Desde esta perspectiva -no superada del todo hoy- la calidad de la educación superior suponía la existencia de estudiantes y académicos sobresalientes, y aseguramientos materiales de primer nivel. Obviamente, esta concepción está a tono con la consideración del carácter elitista de la educación superior y se contrapone a la idea de la educación, incluso la superior como derecho humano.

La misma no solo no ha sido superada del todo sino que es predominante aun en algunos escenarios, aunque ha ido cambiando a partir de las transformaciones en los criterios predominantes sobre la relación universidad sociedad y las exigencias de la sociedad a la educación superior; hoy se hace referencia a un nuevo rol de las universidades en la generación de conocimientos, reconociendo la necesidad y las posibilidades de co-generar el conocimiento junto a otros actores sociales, y por consiguiente la necesidad de formar profesionales preparados para emprender esta tarea; en este sentido, la calidad de la educación superior se enfoca como respuesta a los requerimientos de la sociedad en que se inserta, y se define fundamentalmente sobre la base de su pertinencia social.

La concepción de calidad de la educación superior en Ecuador se corresponde con este enfoque de pertinencia social; en la Ley Orgánica de Educación Superior de la República de Ecuador (LOES) se reconoce el principio de la calidad como uno de los principios de la misma; en su artículo 93 se define la calidad como "...la búsqueda constante y sistemática de la excelencia, la pertinencia, producción óptima, transmisión del conocimiento y desarrollo del pensamiento mediante la autocrítica, la crítica externa y el mejoramiento permanente." (LOES, 2010)

Precisando la significación de la respuesta a las necesidades sociales como indicador de calidad en el artículo 107 de la ley a que se hace referencia anteriormente se define el principio de pertinencia al señalar que "... consiste en que la educación superior responda a las expectativas y necesidades de la sociedad, a la planificación nacional, y al régimen de desarrollo, a la prospectiva de desarrollo científico, humanístico y tecnológico mundial, y a la diversidad cultural..." (LOES, 2010). 
La calidad del docente deviene un factor muy importante en la calidad de la educación al que es necesario prestar atención especial, el docente junto al estudiante es uno de los actores principales del proceso educativo, su formación debe responder al modelo de sociedad en que desempeña su actividad y sobre todo al deber ser de la misma; en las condiciones del mundo de hoy cada vez más interconectado y caracterizado por la movilidad social -la cual tiene entre sus manifestaciones concretas la movilidad estudiantil y la movilidad académica- la formación de docentes debe responder a las exigencias de la educación y la escuela a nivel planetario.

La problemática de la formación docente ha sido objeto de atención por la Organización de las Naciones Unidad para la educación y la cultura (UNESCO) en diversos foros y documentos. Valorando los aspectos que influyen de manera crítica en la formación de docentes, esta organización considera:

1. El bajo nivel de formación con que se ingresa a los estudios pedagógicos.

2. Débil calidad de los programas y los procesos de formación.

3. La limitada formación de los formadores de docentes.

4. La formación universalista para los grupos sociales desfavorecidos.

5. La tensión entre lógicas escolarizantes y lógicas académicas en la formación docente.

6. La insuficiente regulación de los programas de formación docente.

Según Edgar Morin (27) los siete saberes necesarios a la educación del futuro que la escuela tiene por misión enseñar hoy, para dar respuesta a las necesidades de la sociedad contemporánea son:

1. Las cegueras del conocimiento: el error y la ilusión. Que fundamentan la necesidad de promover un conocimiento capaz de aprehender los problemas globales y fundamentales e insertar en ellos los parciales y locales.

2. Los principios de un conocimiento pertinente. Entendido como un conocimiento capaz de aprehender la realidad en su contexto, en su complejidad, teniendo en cuenta las relaciones mutuas y las influencias recíprocas entre las partes y el todo.

3. Enseñar la condición humana. Sobre la base de la unidad compleja de la naturaleza humana la cual debe ser reconocida y abordada integralmente por la ciencia para evidenciar la unidad y diversidad de lo humano en su unidad.

4. Enseñar la identidad terrenal. Demostrando el destino común de los seres humanos, que confrontan los mismos problemas.

5. Afrontar las incertidumbres. Incluir en la enseñanza las incertidumbres y enseñar estrategias para enfrentar los imprevistos, lo inesperado y la incertidumbre y modificar su desarrollo.

6. Enseñar la comprensión. Reconociendo la importancia de la educación para la comprensión como una de las bases más seguras para la educación para la paz.

7. La ética del género humano. Que permita tomar conciencia de Tierra-Patria y que esta conciencia se traduzca en voluntad de realizar la ciudadanía terrena.

Los maestros y profesores capaces de enseñar estos saberes deben tener una formación amplia que les permite no solo adquirir conocimientos relacionados con las Ciencias Pedagógicas y de la Educación, sino poseer una amplia cultura y una profunda formación ético axiológica.

De ahí que los docentes hoy deben formarse:

- En el dominio de los recursos de información y de habilidades sociales, cognitivas y lingüísticas que les permitan adaptarse críticamente a los cambios.

- En valores morales para contribuir al desarrollo de sociedades más justas.

- En el trabajo en equipo con otros docentes y profesionales para la elaboración de proyectos educativos coherentes que den continuidad en la familia y la sociedad en general a la labor educativa de la escuela.

- En la innovación para mejorar su práctica a través de procesos de investigación desde la propia acción.

- En la atención a la diversidad, para trabajar con niños y niñas procedentes de diferentes contextos sociales y culturales con diferentes capacidades, ritmos y estilos de aprendizaje.

Ante estos retos cabe preguntarse ¿Quiénes ejercen mayoritariamente la profesión docente en América Latina hoy? La mayoría mujeres sometidas por tanto a discriminación y abusos, personas generalmente de posición socioeconómica relativamente baja y en algunos países con un considerable por ciento de envejecimiento. ¿Son atraídos a la profesión los jóvenes con mayor vocación y talento? 
En la mayoría de los casos no ¿Poseen altos estándares de calidad las instituciones y programas de formación? La respuesta es también negativa, si se hace referencia a la educación pública. La mayor parte de los profesores aprenden a serlo por ensayo y error, en las peores condiciones laborales posibles, y con escasa formación útil para enfrentar los nuevos retos de la enseñanza con un trabajo de calidad.

Al referirse al estado actual de esta problemática el Informe del Grupo del Banco Mundial. Profesores excelentes: Cómo mejorar el aprendizaje en América Latina y el Caribe señala "...que la calidad de los profesores de América Latina y el Caribe es la limitación más importante al avance de la región hacia sistemas educativos de calidad internacional..." (Banco Mundial, 2014) y al valorar los diferentes factores que influyen el mejoramiento de la calidad del personal docente destaca el proceso de formación.

La calidad de la formación se utiliza define un conjunto de cualidades del proceso de formación que resultan de la interrelación de la excelencia académica y la pertinencia integral, entendida esta última en su acepción más amplia; la misma se asocia fundamentalmente a la calidad de los recursos humanos, de la base material y de la gestión del proceso de formación. Esta constituye un proceso que implica el mejoramiento continuo a partir del control de la calidad, en el que se involucran todas las personas e instancias vinculadas al proceso de formación y especialmente los docentes.

¿Quiénes ejercen mayoritariamente la profesión docente en Ecuador hoy? ¿Cuál es el estado de las instituciones y los planes de formación docente? La respuesta a estas interrogantes exige estudios científicamente fundamentados, no obstante, los autores de este artículo comparten el criterio de que la formación de docentes en Ecuador no se encuentra aún en condiciones de cumplir con las exigencias de la educación en el siglo XXI.

¿Dónde se forman los docentes ecuatorianos? La formación de maestros ha tenido en el país un largo proceso, en un primer momento encargó a los colegios normales; a partir de 1991 se constituyen en institutos pedagógicos, instituciones dedicadas a la formación docente y a la investigación aplicada articuladas académicamente a la Universidad Nacional de Educación (UNAE) que forman profesores de educación primaria, aunque su competencia cubre también el nivel pre-primario. En las facultades de educación, que dependen de universidades ubicadas en las diferentes provincias del país; y que se agrupan en la Asociación de Facultades Ecuatorianas de Filosofía, Letras y Ciencias de la Educación.

Sin embargo, no todos los que ejercen la profesión tienen formación docente ya que debido alto déficit de docentes; se ha tenido que recurrir a personas sin formación especializada, entre ellas, personas con títulos de bachilleres o universitarios.

Un aspecto importante que incide en el proceso de formación es la cantidad y calidad del ingreso, en los últimos tiempos se ha manifestado una tendencia decreciente en el ingreso a las carreras pedagógicas debido a diversas causas, entre las que se encuentran: falta de reconocimiento social de la profesión, salarios bajos de los docentes, creciente migración de la población joven, altos índices de pobreza presente en los hogares ecuatorianos, falta de conciencia sobre la importancia del maestro e insuficiencias en la formación que inciden negativamente en la motivación hacia la profesión docente.

El Dr. Villarroel Idrovo, Jorge. (2017) refiriéndose a la formación docente en Ecuador considera que “... el currículo de la formación docente en el país, y hasta en el mundo entero, ha sido un fracaso "independientemente de que desde el criterio de los autores de este artículo tal afirmación resulta muy categórica y lapidaria, se comparten algunas de las ideas con las que este autor argumenta sus posiciones.

Según su criterio, el currículo se ha caracterizado por el academicismo (centrado en contenidos teóricos, disciplinares y desestimando otras facetas de la formación integral como valores, sentimientos, actitudes, cultura, ideología); el cientificismo (definido desde comunidades académicas especializadas, con el propósito de formar para y desde la ciencia); la disciplinariedad (que atomiza el conocimiento humano en disciplinas sin conexiones entre ellas).

El enciclopedismo (gran cantidad de contenidos y materias); la descontextualización (no se evidencia la aplicabilidad de los contenidos que aprenden a la solución de problemas profesionales); orientado a la reproducción del conocimiento; el positivismo (la visión dominante de la ciencia que se transmite tiene un enfoque positivista);que privilegia el instruir por sobre el educar y descuida la responsabilidad social del docente; monocultural (en un paradójicamente país intercultural); desideologizado; que predomina la medición y descuida la investigación y que por tanto considera antidemocrático, eurocentrista y que contribuye al analfabetismo funcional. 
Es necesario esclarecer, que las insuficiencias en la formación no son atribuibles solo al currículo, el cual presenta las mayores dificultades generalmente en su implementación, y no en su concepción.

\section{Exigencias y retos para la educación ecuatoriana}

En las condiciones de hoy y para dar respuesta efectiva a las exigencias del mejoramiento continuo de la calidad de la educación superior la formación continua del docente debe centrarse en su práctica educativa y encaminarse a:

- Lograr mayor vínculo entre la formación inicial y la práctica.

- Asimilar cultura de la profesión, incluyendo una cultura de la calidad y de la evaluación y acreditación de la calidad.

- Actualizar el currículo y lograr el mejoramiento de la calidad y equidad educativa

- Ayudar a grupos diversos de profesores a desarrollar competencias, fundamentalmente pedagógicas, didácticas e investigativas.

- Instalar una cultura de la evaluación, coherente con el concepto de calidad de la educación que parta del desarrollo de la autoevaluación.

- Asimilar las nuevas características de la docencia y el replanteo de las funciones del docente como resultado de las influencias de las tecnologías de la información y las comunicaciones.

- Desarrollar y mejorar sistemas de evaluación de la formación y el desempeño docente.

Estas exigencias y retos se contextualizan de manera particular en la carrera de Educación General Básica (EGB) la cual tiene como centro del proceso de enseñanza-aprendizaje al estudiante, como un sujeto ético, innovador y transformador de la realidad educativa y como objetivo formar profesionales con compromiso ético, docentes de excelencia capacitados para investigar, analizar, planificar, evaluar y retroalimentar planes, programas, proyectos y estrategias educativas y curriculares. Esta carrera forma docentes para la Educación General que comprende a los niños/as y adolescentes desde los 5 años de edad hasta el Bachillerato

El egresado de esta carrera, deberá estar preparado para reconocer y valorar las diversas formas del saber y sus expresiones particulares en relación a las problemáticas socioculturales y ambientales de su contexto; analizar los núcleos de la pedagogía como ciencia y de las disciplinas relacionadas; interpretar los fundamentos filosóficos, epistemológicos y culturales del aprendizaje humano y sus implicaciones en los sistemas de enseñanza e investigar para aprender sobre los procesos de aprendizaje.

\section{CONCLUSIÓN}

La calidad de la educación depende en gran medida de la calidad del personal docente debido al papel que este desempeña y a las transformaciones en su rol profesional como consecuencia de las transformaciones sociales. En Latinoamérica en general, y en Ecuador en particular, la formación de docentes presenta insuficiencias que inciden directa y negativamente en la calidad de la educación por lo que ante las políticas públicas en general y las educativas en particular se presentan importantes retos.

El desarrollo de políticas integrales para el perfeccionamiento de la formación docente, y, consiguientemente del ejercicio de la profesión docente, deviene condición indispensable para enfrentar los retos actuales, políticas sistémicas, integrales e intersectoriales que permitan crear y fortalecer sistemas articulados de formación continua y fortalecer el papel de la escuela como centro para la formación, el desarrollo profesional y la evaluación docente.

\section{BIBLIOGRAFÍA}

1. Abendaño Briceño, Augusto (2004) Procesos de Formación de los Docentes por parte de Universidades e Institutos Pedagógicos en Ecuador. Digital observatory for higher education in Latin America and the Caribbean www.iesalc.unesco.org.ve

2. Constitución de la República del Ecuador (2008). Recuperado de http://www.cicad.oas.org/fortalecimiento_institucional/legislations/PDF/EC/constitucion.pdf

3. Grupo del Banco mundial. Profesores excelentes: Cómo mejorar el aprendizaje en América Latina y el Caribe. Washington, DC ,2014

4. Fabara Garzón Eduardo. (2017) La formación para la docencia en el Ecuador.En La formación y el trabajo del docente en Ecuador. 1era. Edición: Universidad Politécnica Salesiana. ISBN: 978-9978-10-276-3 Editorial Abya-Yala Quito-Ecuador 
5. Morin, E. (2000). Los siete saberes necesarios a la educación del futuro. Caracas, Venezuela: Ediciones FACES. IE SALC- UNESCO.

6. Organización de Naciones Unidas (2015). Objetivos de Desarrollo del Milenio Informe de 2015.http://www.un.org/es/millenniumgoals/pdf/2015/mdg-report-2015_spanish.pdf

7. Presidencia de la República. Ley Orgánica de Educación Superior (2010). http://www.yachay.gob.ec/wpcontent/uploads/downloads/2013/12/LEY-ORGANICA-DE-EDUCACION-SUPERIOR-ANEXO-a_1_2.pdf

8. Registro Oficial (2011). "Ley Orgánica de Educación Intercultural” Asamblea Nacional. Quito, Ecuador.

9. UNESCO (2013). Elaboración de Políticas Docentes en América Latina y el Caribe”. Santiago, Chile: Oficina Regional de Educación para América Latina y el Caribe (OREALC).

10. Villarroel Idrovo, Jorge. (2017) El fracaso de la formación docente. En La formación y el trabajo del docente en Ecuador. 1era. Edición: Universidad Politécnica Salesiana. ISBN: 978-9978-10-276-3Editorial Abya-Yala QuitoEcuador 